\title{
A SET OF FOUR INDEPENDENT POSTULATES FOR
}

\section{BOOLEAN ALGEBRAS*}

\author{
BY
}

\section{B. A. BERNSTEIN}

In these Transactions, October, 1913, Sheffer $\dagger$ presented the following set of independent postulates for Boole's logic:

I. There are at least two distinct $K$-elements.

II. Whenever $a$ and $b$ are $K$-elements, $a \mid b$ is a $K$-element.

Definition. $a^{\prime}=a \mid a$.

III. Whenever $a$ and the indicated combinations of $a$ are $K$-elements, $\left(a^{\prime}\right)^{\prime}=a$.

IV. Whenever $a, b$, and the indicated combinations of $a$ and $b$ are $K$ elements, $a \mid\left(b \mid b^{\prime}\right)=a^{\prime}$.

$\mathrm{V}$. Whenever $a, b, c$, and the indicated combinations of $a, b$, and $c$ are $K$-elements, $[a \mid(b \mid c)]^{\prime}=\left(b^{\prime} \mid a\right) \mid\left(c^{\prime} \mid a\right)$.

This is the most economical set of postulates that has so far been proposed for Boolean algebras. Not only is the number of primitive propositions considerably smaller than that of the smallest set $\ddagger$ of previous date-five instead of nine-but also the special elements "zero," the "whole," and the "negative" are all defined and their properties deduced. This economy Dr. Sheffer effected by basing the algebra on the powerful operation of "rejection," $\mid . \S$ Choosing the primitive ideas that Dr. Sheffer chose, I give below a set of four independent postulates from which his five Postulates $\mathrm{I}-\mathrm{V}$ are easily deduced.

NeW SET OF FOUR POSTULATES FOR BOOLEAN LOGIC

If we take class $K$ and operation | as primitive ideas, || Boolean logic can be deduced from the following postulates:

* Presented to the Society, August 3, 1915.

$\dagger$ H. M. Sheffer: $A$ set of five independent postulates for Boolean algebras, with application to logical constants, these T r a n s a c t i o n s, vol. 14 (1913), pp. 481-488.

$\ddagger$ Professor E. V. Huntington's. See his Sets of independent postulates for the algebra of logic, these Transactions, vol. 5 (1904), pp. 288-309. See also the writer's $A$ complete set of postulates for the logic of classes, etc., $\mathrm{U} n$ iversit y of $\mathrm{C}$ a l if or $\mathrm{nia} \mathrm{Pub}$ lications in $M$ athematics, vol. 1, no. 4, pp. 87-96.

$\S$ Interpreted concretely, the "reject" $a \mid b$ may be read: "that which is neither $a$ nor $b . "$

$\|$ The symbol " $=$ " is not taken as a primitive idea. By $a=b$, we agree to mean that $a$ and $b$ can be interchanged. 
$P_{1} . K$ contains at least two distinct elements.

$P_{2}$. If $a, b$ are elements of $K, a \mid b$ is an element of $K$.

Definition 1. $a^{\prime}=a \mid a$.

$P_{3}$. If $a, b$, and the combinations indicated are elements of $K$,

$$
(b \mid a) \mid\left(b^{\prime} \mid a\right)=a \text {. }
$$

$P_{4}$. If $a, b, c$, and the combinations indicated are elements of $K$,

$$
a^{\prime} \mid\left(b^{\prime} \mid c\right)=\left[\left(b \mid a^{\prime}\right) \mid\left(c^{\prime} \mid a^{\prime}\right)\right]^{\prime} .
$$

The following system satisfies all the Postulates $P_{1}-P_{4}$, and thus proves these postulates to be consistent:

$K=$ a class of two elements $e_{1}, e_{2} ; e_{i} \mid e_{j}=$ the element given by the following "rejection" table,

Theorem 1. $\left(a^{\prime}\right)^{\prime}=a$.

$$
\begin{array}{c|cc}
\mid & e_{1} & e_{2} \\
\hline e_{1} & e_{2} & e_{1} \\
e_{2} & e_{1} & e_{1}
\end{array}
$$

For, in $P_{4}$, let $b=c=a$. Then, by Definition 1 and $P_{3},{ }^{*}$ the left member becomes

$$
a^{\prime}\left|\left(a^{\prime} \mid a\right)=(a \mid a)\right|\left(a^{\prime} \mid a\right)=a ;
$$

and, by $P_{3}$, the right member becomes

$$
\left[\left(a \mid a^{\prime}\right) \mid\left(a^{\prime} \mid a^{\prime}\right)\right]^{\prime}=\left(a^{\prime}\right)^{\prime} .
$$

Definition 2. $a^{\prime \prime}=\left(a^{\prime}\right)^{\prime}$.

Theorem 2. $a \mid\left(b \mid b^{\prime}\right)=a^{\prime}$.

For, by $1, P_{4}, 1$, and $P_{3}, \dagger$

$a\left|\left(b \mid b^{\prime}\right)=a^{\prime \prime}\right|\left(b^{\prime \prime} \mid b^{\prime}\right)=\left[\left(b^{\prime} \mid a^{\prime \prime}\right) \mid\left(b^{\prime \prime} \mid a^{\prime \prime}\right)\right]^{\prime}=\left[\left(b^{\prime} \mid a\right) \mid\left(b^{\prime \prime} \mid a\right)\right]^{\prime}=a^{\prime}$.

Theorem 3. $[a \mid(b \mid c)]^{\prime}=\left(b^{\prime} \mid a\right) \mid\left(c^{\prime} \mid a\right)$.

For, by $1, P_{4}, 1,1, \dagger$

$$
\begin{aligned}
{[a \mid(b \mid c)]^{\prime}=\left[a^{\prime \prime} \mid\left(b^{\prime \prime} \mid c\right)\right]^{\prime}=\left[\left(b^{\prime} \mid a^{\prime \prime}\right) \mid\left(c^{\prime} \mid a^{\prime \prime}\right)\right]^{\prime \prime} } & \\
& =\left[\left(b^{\prime} \mid a\right) \mid\left(c^{\prime} \mid a\right)\right]^{\prime \prime}=\left(b^{\prime} \mid a\right) \mid\left(c^{\prime} \mid a\right) .
\end{aligned}
$$

Since Sheffer's Postulates I, II are identical with Postulates $P_{1}, P_{2}$, and since Postulates III, IV, V correspond respectively to Theorems 1, 2, 3,-all of Sheffer's postulates, and hence all of Boolean logic, can be deduced from Postulates $P_{1}-P_{4}$. Indeed, the two sets of postulates are equivalent; for propositions $P_{1}-P_{4}$ can also be derived from $\mathrm{I}-\mathrm{V}$, as may be easily verified.

* The use of $P_{1}$ and $P_{2}$ will not be indicated.

$\dagger$ The use of Definition 2 is not indicated. 


\section{INDEPENDENCE OF THE FOUR POSTULATES}

The following systems $\bar{P}_{1}-\bar{P}_{4}$ are such that $\bar{P}_{i}$ contradicts none of the Postulates $P_{1}-P_{4}$ except $P_{i}$, thus showing the independence of $P_{i}$ from the rest of the postulates.

$\bar{P}_{1} . K=$ a class of one element $e ; e \mid e=e$.

$\bar{P}_{2} . K=$ a class of two elements $e_{1}, e_{2} ; e_{i} \mid e_{j}$ is given by the following table:

$$
\begin{array}{c||ll}
\frac{e_{1}}{e_{1}} & e_{2} \\
\hline e_{2} & x
\end{array}
$$

where $x$ is not in $K . \quad P_{2}$ is false for $a \neq b$.

$\bar{P}_{3} . K=$ a class of two elements $e_{1}, e_{2} ; e_{i} \mid e_{j}$ is given by the following table:

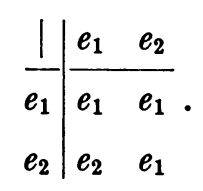

Here $P_{3}$ is false for $a=e_{1}, b=e_{2}$.

$\bar{P}_{4} . K=$ a class of two elements $e_{1}, e_{2} ; e_{i} \mid e_{j}$ is given by the following table:

$$
\begin{array}{l|ll}
\frac{e_{1}}{e_{1}} & e_{2} \\
\hline e_{2} & e_{2} \\
e_{1} & e_{2}
\end{array}
$$

Here $P_{4}$ is false for $a=e_{1}, c=e_{2}$.

University of California,

May, 1915 